\title{
oints
}

$\sqrt{2}$

\section{A future in our past: the umbilical cord for orthopaedic tissue engineering}

\author{
ANTONIO MARMOTTI', GIUSEPPE MARIA PERETTI², SILVIA MATTIA ${ }^{3}$, DAVIDE EDOARDO BONASIA ${ }^{1}$, \\ MATTEO BRUZZONE ${ }^{1}$, FEDERICO DETTONI ${ }^{1}$, ROBERTO ROSSI ${ }^{1}$, FILIPPO CASTOLDI ${ }^{1}$ \\ ${ }^{1}$ University of Turin, Department of Orthopaedics and Traumatology, Turin, Italy \\ ${ }^{2}$ Department of Biomedical Sciences for Health, University of Milan, Milan, Italy, IRCCS Istituto Ortopedico Galeazzi, \\ Milan, Italy \\ ${ }^{3}$ University of Turin, Molecular Biotechnology Center, Turin, Italy
}

\begin{abstract}
The umbilical cord (UC) has recently been added to the list of potential cell sources for tissue engineering and regenerative medicine purposes. Although the UC is usually discarded after delivery, UC storage in special tissue banks is becoming an increasingly common procedure.

Indeed, the capacity of UC cells to be directed toward different phenotypes makes this tissue an ideal cell source for regenerative medicine in orthopedics and in other fields. In this paper, these issues are presented and discussed, together with the potential of this cell source for allogeneic use. This article also looks at the anatomy of the UC from both the macroscopic and the cellular perspective and considers its extraordinary potential for research and clinical applications.
\end{abstract}

Key Words: chondrogenic, osteogenic, mesenchymal stem cells, tissue engineering, umbilical cord.

"Time present and time past

Are both perhaps present in time future,

And time future contained in time past."

(Burnt Norton, T.S. Eliot: Four Quartets, 1935)

We often need to look to our past that to find the solu-

\section{Corresponding Author:}

Antonio Marmotti, MD

Department of Orthopaedics and Traumatology,

University of Turin Medical School,

Mauriziano "Umberto I" Hospital

Largo Turati 62, 10128 Turin, Italy

Phone: +39-3388308700

E-mail: antonio.marmotti@inwind.it tions and strength we need in our present and for our future life; sometimes our past, helping us to find our meaning, allows us to shape our future as an unexpected original composite of our time present and our time past. The force of these fascinating literary concepts is such that they extend beyond the bounds of pure philosophy and seem to suggest a direction for research in different scientific fields: one of these is tissue engineering.

Certainly, these are insights that seem to fit perfectly with the growing interest in the use of the umbilical cord (UC) as a source of stem cells.

The UC is our primary link to external space; it is also the fetal structure that supplies us with our first energy for living. Indeed, during pregnancy, the fetus is linked to the mother and her placenta by the UC, which envelops and protects umbilical vessels and provides cord blood to the fetus. The UC contains two umbilical arteries, an umbilical vein and a mucous proteoglycan-rich connective tissue, named Wharton's jelly after Thomas Wharton who first described it in 1656, covered by amniotic epithelium. Thus, five regions can be identified, each containing cells with features similar to those of the mesenchymal stem cells (MSCs) of other compartments of the human body. MSCs can be isolated from mononuclear cell fractions, from UC blood, from the subendothelial layer of the umbilical vein, from the outer layers of umbilical vessels (the perivascular region), from the intravascular space and from the subamnion region.

The efficiency of cord blood, which seems to contain a small amount of mesenchymal precursor cells, is hampered by the low quantity of blood obtainable and a low success rate of isolation. Literature data suggest that the 
frequency of circulating MSCs in cord blood is approximately $0.002 \pm 0.004$ per $10^{6}$ initially plated cells, while the number of colony forming units-fibroblasts (CFUF) from a "classical" stem cell source like the bone marrow $(\mathrm{BM})$ can be estimated as $83 \pm 61$ per $10^{6}(1)$.

Using the "solid" part of the UC is, on the other hand, a completely different issue. The UC is collected during cesarean birth, to allow the best possible conditions in terms of sterility, and a large amount of tissue can be collected: in our experience, a mean of $32 \mathrm{~g}$, corresponding to a mean length of $37 \mathrm{~cm} \mathrm{(2).}$

The first aspect to consider in relation to this procedure is the fact that this harvested living tissue was formerly material that was simply discarded at the end of a delivery. As a consequence, the use of this extraembryonic structure, whose cells possess some embryonic properties and considerable regenerative potential, in theory presents few ethical problems and legal concerns, provided that complete and informed written consent is obtained from the donating mother. This aspect is fundamental from the perspective of the possible widespread clinical application of the UC in the field of tissue engineering in the future.

A second advantage, soon recognized by researchers, is the unlimited availability of this tissue. Tissue banks for the collection and storage of cells from UCs donated by third parties are a conceivable future reality for bone and cartilage tissue engineering based on the use of "one-stage" cell delivery methods (scaffolds or intraarticular injections).

Finally, other non-negligible aspects are the non-invasiveness and low costs of the UC harvesting and cell isolation procedures. Indeed, neither UC collection from mothers nor, logically, the receipt of UC-derived cells of allogeneic origin by target patients, involves donor site morbidity. This is an appealing advantage compared with other sources of stem cells, such as BM or adipose tissue. Indeed, BM presents specific drawbacks, which limit its usefulness: cell isolation requires aspiration from the marrow cavity, which is a painful and invasive procedure that carries certain risks. Moreover, adult-derived MSCs may have limited expansion potential and show slower expansion in vitro compared with fetal-derived MSCs. Indeed, they can be defined "age-dependent" as they show a decrease in yield and proliferative capacity with increasing decades of life. On the other hand, the UC, by definition "deriving from" a perinatal period, always retains some embryonic properties.

Once obtained, UC tissue has to be processed to extract the cells. This step has been widely studied in the literature and different methods have been proposed (1). Enzymes such as collagenase I, trypsin and hyaluronidase have been employed in quite complex protocols that involve manipulation of the original tissue. These procedures claim to improve the "purity" of the Wharton's jelly cell population, eliminating most of the endothelial precursor cells that reside strictly in the perivascular space (i.e. cells from the umbilical vein subendothelium). This approach is at the very least questionable, given that the strength of the UC as a reservoir of precursor cells lies precisely in the fact that it contains cells showing different phenotypic profiles (this applies not only to cells coming from the various parts of the cord, but also to cells originating from the same UC regions). It has been observed that cells located close to the amniotic surface display an enhanced ability to proliferate, whereas more differentiated cells have been found in closer proximity to the umbilical vessels. This suggests that precursor cells from the UC are a peculiar cell family whose individuals show various degrees of stemness, rather as different children from the same family may have different personalities. Accordingly, a method of cell harvesting that involves no enzymatic digestion or major manipulation is preferable. We, in our research, used a modification of the La Rocca procedure (3), based on the "mesenchymal" migratory capability shown by precursor cells from the UC. This raises strong analogies with other research fields that deal with tissue repair using different precursor cells, such as the migrating chondrocytes from minced cartilage fragments (4). In brief, UC tissue is minced manually into very small cuboidal fragments (4-7 mm length) (Fig. 1) and these small UC pieces are transferred to Petri dishes where they are evenly distributed and incubated in the MSC expansion medium for up to two weeks. Thereafter, the UC tissue is removed and adherent cells are allowed to expand; finally, after an intermediate passage, cells are retrieved from the dishes at approximately 42 days.

The population obtained using our protocol usually 


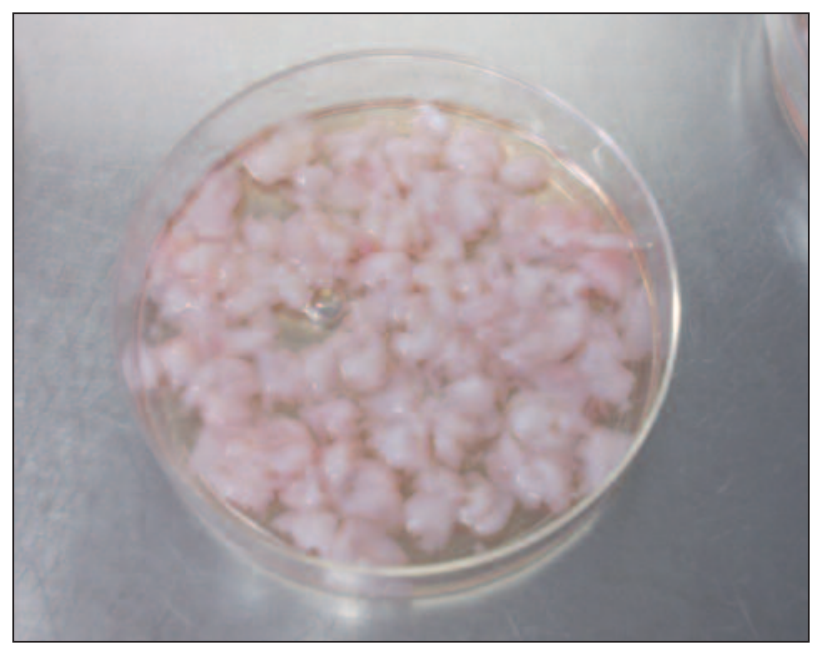

Fig. 1. Fragments of umbilical cord in a Petri dish.

shows great efficiency, because for each gram of original UC tissue, $0.8 \times 10^{6}$ cells are retrieved (5).

As reported in the literature (6), UC cells may be considered "mesenchymal" because they follow the minimal criteria proposed in 2006 by the Mesenchymal and Tissue Stem Cell Committee of the International Society for Cellular Therapy. Indeed, multipotent mesenchymal stromal cells have to respect three criteria: adherence to plastic culture, specific surface antigen expression (CD105, CD73, CD90 and minimal expression of CD45, CD34, CD14 or CD11b, CD79a or CD19, STRO-1 and HLA class II proteins), and multipotent differentiation potential along osteogenic, adipogenic and chondrogenic lineages.

As regards surface antigen expression, we have observed, following use of our extraction method, a notable presence $(40 \%)$ of double negative cells, i.e. negative for both HLA-ABC and HLA-DR proteins (7). These cells may represent a particular subpopulation with appealing features for allogeneic use. Moreover, recent literature data suggest that UC-MSCs, like embryonic stem cells, express pluripotency markers Oct-4, Sox-2 and Nanog (6). This underlines the peculiar embryonic properties of UC-MSCs, showing them to be a "bridge" between embryonic and adult stem cells, being "more primitive" than those found in BM and representing an earlier-stage mesenchymal-like stem cell category than that deriving from adult fat or BM. These peculiar aspects introduce the concept of the immunoprivileged status of UC-MSCs. Besides the low expression of MHC class I antigens and the low or null expression of MHC class II antigens, at variance with BM-MSCs which are "less privileged", these cells also express the HLA-G6 isoform (8). The low levels of MHC class I antigens may protect them against attack by natural killer cells, while HLA-G6 is involved in immunomodulation during pregnancy, preventing a maternal immune response against the fetus; HLA-G6 may also be involved in other mechanisms of immunotolerance, as it may promote the expansion of regulatory $\mathrm{T}$ cells contributing to the suppression of effector responses to alloantigens. Moreover, UCMSCs produce IL-10, TGF-beta, IL-6, VEGF, prostaglandin E2 (PGE2), cyclooxygenases (COX), hepatocyte growth factor (HGF), human leukocyte antigens G5 and E, leukemia inhibitor factor, and indoleamine 2,3-dioxygenase (IDO), all of which are related to the immunosuppressive capability of MSCs. On this basis, it can be hypothesized that the immune properties of UC-MSCs are expressed at multiple levels, through: low stimulation of allogeneic T-lymphocyte proliferation, suppression of activated $\mathrm{T}$ lymphocytes, increased regulatory $\mathrm{T}$-cell production, and reduced Bcell stimulation. These actions are likely achieved both through a direct, contact-dependent mechanism, by means of the expression of the cell death ligand B7$\mathrm{H} 1$, and a contact-independent mechanism mediated by cytokines and growth factors. The latter can be clearly observed when culturing $\mathrm{T}$ cells in the presence of UC-MSC supernatant, which is able to inhibit T cell proliferation after 5 and 7 days, compared with the RPMI-1640 medium supplemented with phytohemagglutinin and IL-2 (5). In view of these considerations, it is unsurprising that the clinical-experimental use of UC-MSCs against particular forms of severe steroid-resistant acute graft-versus-host disease is already a reality (9).

Along with these immune properties, another feature common to UC-MSCs and other MSC counterparts of different origins is their multiple differentiation capacity. There indeed exists extensive in vitro evidence that they show osteogenic, adipogenic and pellet chondrogenic commitment $(7,10)$. Moreover, a potential to differentiate toward other cell lines has been widely exploited and it is well known that UC-MSCs are capable of differentiating toward heart cells (cardiomyocy- 
tes), skeletal muscle cells, endothelial cells (10), neural cells, cells expressing markers of hepatic lineage, and isle-like cells able to express pancreatic associated genes (11). Some peculiar aspects of this behavior are worth commenting on in more detail.

First, the commitment of UC-MSCs seems to be influenced by the perinatal environment; for example, it has been observed that UC-MSCs from diabetic mothers show higher adipocyte differentiation efficiency than that shown by MSCs from healthy mothers (12). This seems to indicate that the uterine microenvironment plays a role in regulating the different behavior of UC-MSCs in the general population and, more specifically, in influencing the prevalence of obesity in diabetes by means of a preferential "precommitment" of precursor cells toward adipocytes during the childhood.

Second, adipogenic differentiation seems to be slightly different in UC-MSCs. Lipid accumulation has been observed to take longer in UC-MSC cultures and the resulting adipocytes are less mature than those derived from BM-MSCs (11); we have observed that UCMSC cultures showed smaller lipid vacuoles, possibly related to brown fat commitment, at variance with BM-derived adipocytes, which showed larger vacuoles (7). This supports a more "embryonic" behavior of UC-MSCs compared with the behavior of their BMderived counterparts.

Third, some studies have shown that UC-MSCs have weaker osteogenic and chondrogenic potential than do BM-MSCs (11).

The idea that UC-MSCs show limited osteogenic commitment is widely debated in the literature. Data from our experience, along with previously published studies, seem to question this limitation in UC-MSC behavior, whether cells are cultured in a monolayer (7) (Fig. 2), grown as cultures on a $3 \mathrm{D}$ scaffold $(2,13)$ (Fig. 3) or loaded onto scaffolds and implanted in a dorsal subcutaneous pouch in nude mice (14).

The hypothesis that UC-MSCs show weak chondrogenic commitment is even more debatable. Some have observed that UC-MSCs, compared with BM-MSCs, are characterized by superior extracellular matrix synthesis in terms of glycosaminoglycans (GAGs) and collagen I production, consistent with a "fibrocartilaginous" commitment, while BM-MSCs seem to be
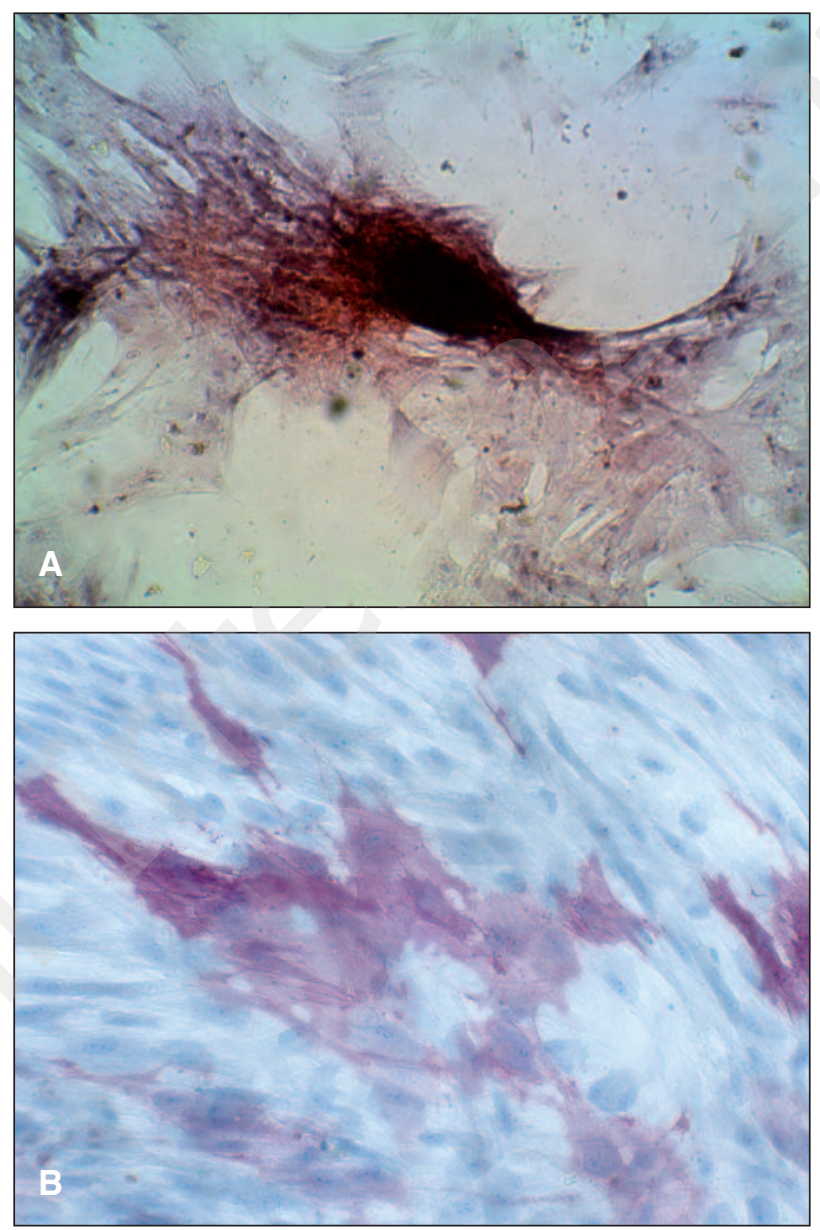

Fig. 2. Osteogenic differentiation of UC-MSCs demonstrated by alizarin red staining $(A)$ and alkaline phosphatase staining $(B)$ at 21 days. Magnification 10x.

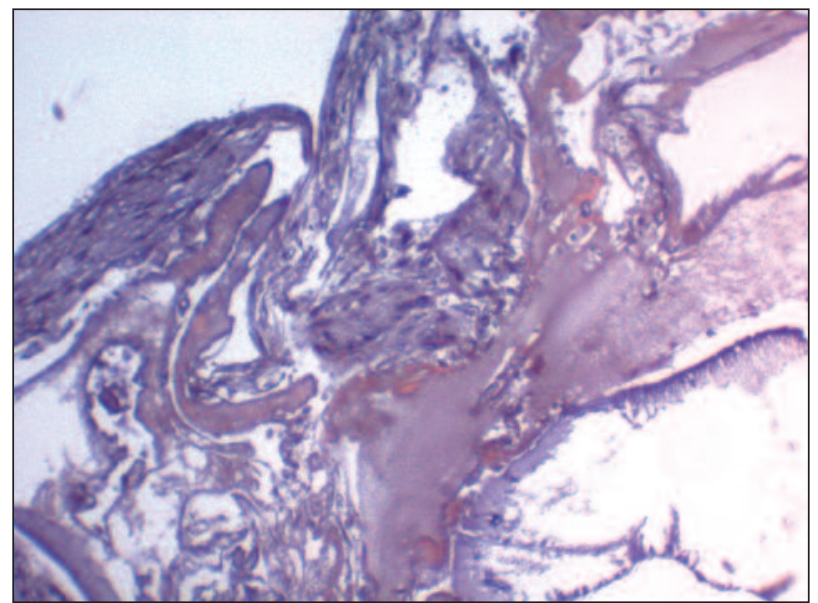

Fig. 3. 3-D culture of UC-MSCs on osteogenic scaffold demonstrated by alizarin red staining (10x) at 30 days. 
more prone to collagen II synthesis and, consequently, to show better chondrogenic differentiation (15). For these reasons, UC-MSCs seem to be good candidates for intervertebral disk regeneration, as shown in different studies $(16,17)$. Even though this preclinical application may provide an intriguing solution to a major problem in the general population, we, on the other hand, still believe that UC-MSCs offer potential for cartilage regeneration. Indeed, data from in vitro pellet cultures (Fig. 4), in vitro high-density cultures on rotatory systems (18) and in vitro 3D cultures on chondrogenic scaffolds (namely collagen I/III and hyaluronic acid derivative) (Fig. 5) have recently demonstrated the fascinating behavior of these cells. They are able to produce chondrogenic markers such as SOX9 and collagen II both in normoxic and hypoxic conditions, as well as produce matrix staining positive for safranin-O (2), and they did not express MHC class II antigens, thereby preserving the immunoprivileged properties of UC-MSCs even in a differentiated condition (18). Moreover, several other studies have
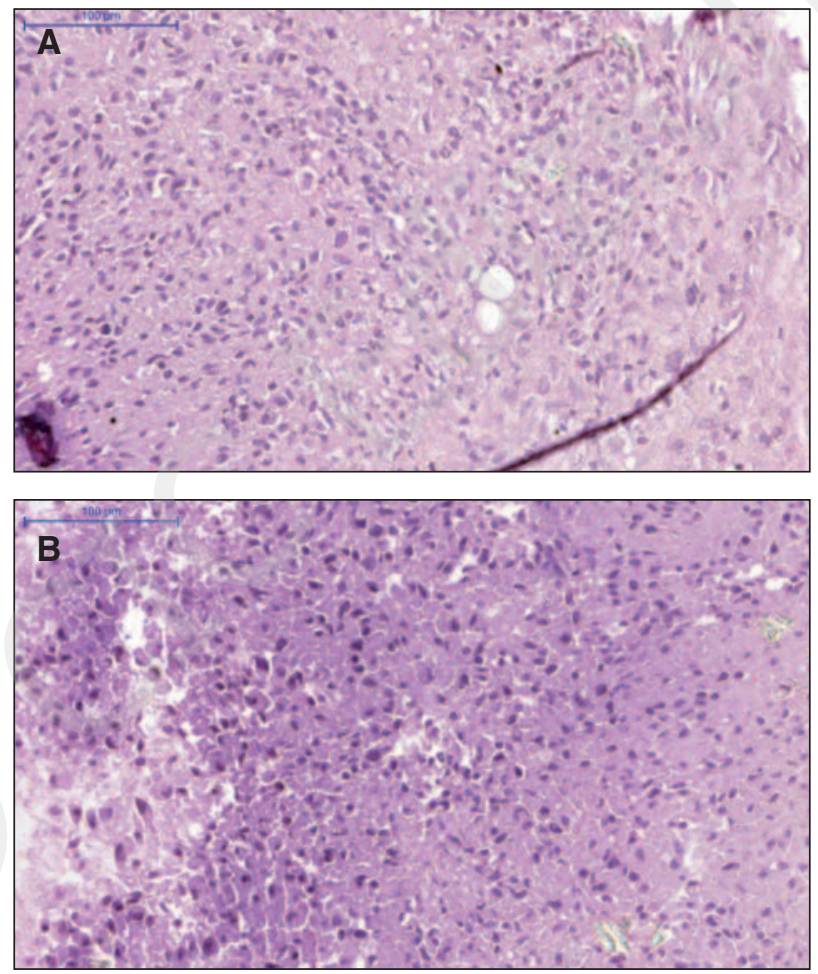

Fig. 4. Pellet culture of UC-MSCs for chondrogenic commitment demonstrated by safranin $\mathrm{O}$ staining at 30 days in normoxic condition (A) and hypoxic condition at $10 \%$ oxygen tension (B).
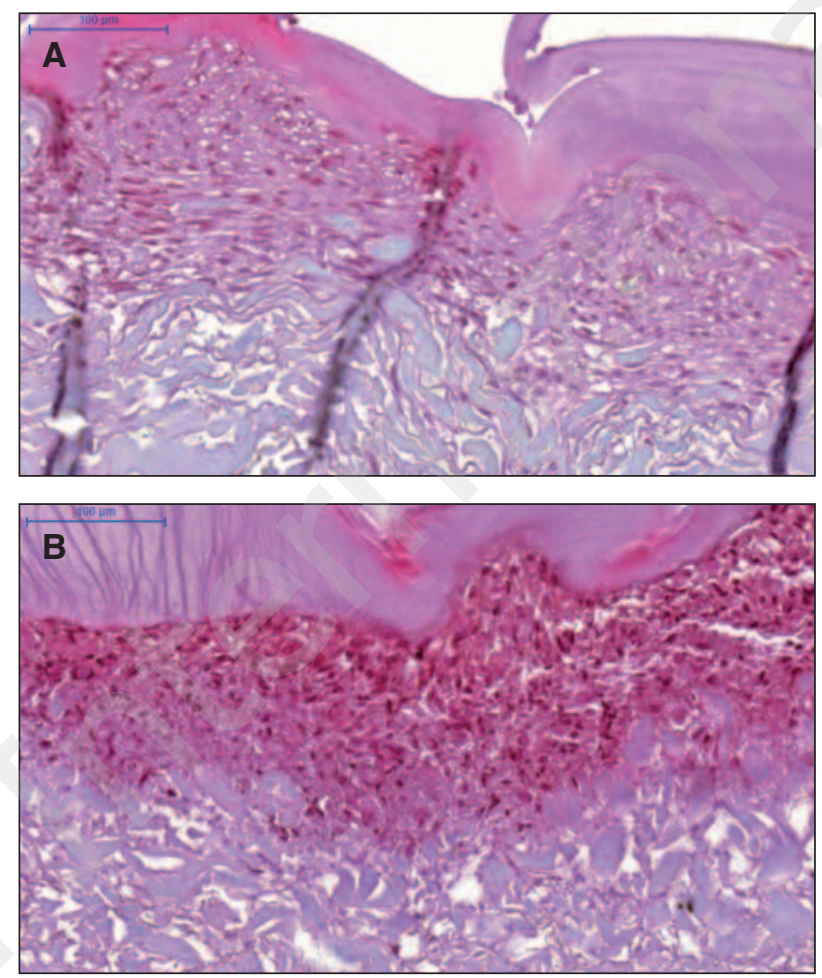

Fig. 5. 3D culture of UC- MSCs on collagen I/III chondrogenic scaffold demonstrated by safranin $O$ staining at 30 days in normoxic condidemonstrated by safranin $(A)$ and hypoxic condition at $10 \%$ oxygen tension (B).
tion

shown marked chondrogenic differentiation of UCMSCs in other, different conditions, e.g. when cells are embedded in a collagen hydrogel (19) or grown on Poly ( $\varepsilon$-caprolactone (PCL)/collagen nanoscaffolds (20). For all these reasons, we believe that UC-MSCs, when combined with suitable scaffolds, could be an attractive potential source for clinical allogeneic use to treat various diseases, including chondral and osteochondral lesions, and may well represent a candidate for "universal off-the-shelf" stem cell products in the field of orthopedic tissue engineering.

Naturally, further studies, both in vitro and at preclinical animal level, are needed to confirm the intuitions gathered in the last few years. Yet, this is certainly not a remote horizon, and the conjoined efforts of basic researchers and clinicians may help to bridge gaps that currently appear difficult to cross.

Ultimately, the simple small umbilical cord could well represent the way forward for orthopaedic allogeneic stem cell therapy. Indeed "Even the smallest thing can change the course of the future." (J.R.R. Tolkien). 


\section{References}

1. Lv F, Lu M, Cheung KMC, Leung VYL, Zhou G. Intrinsic properties of mesemchymal stem cells from human bone marrow, umbilical cord and umbilical cord blood comparing the different sources of MSC. Curr Stem Cell Res Ther. 2012;7:389-399.

2. Marmotti A, et al. e-Poster P133 ICRS 2013 Minced umbilical cord fragments as an effective cell source for cartilage and bone tissue engineering. An in vitro tridimensional study. http://www.cartilage.org/ at <https://www.webges.com/cslide/ library/icrs/mylibrary/search>

3. La Rocca G, Anzalone R, Corrao S, et al. Isolation and characterization of Oct-4+/HLA-G+ mesenchymal stem cells from human umbilical cord matrix: differentiation potential and detection of new markers. Histochem Cell Biol. 2009; 131:267-282.

4. Marmotti A, Bonasia DE, Bruzzone M, et al. Human cartilage fragments in a composite scaffold for single-stage cartilage repair: an in vitro study of the chondrocyte migration and the influence of TGF- 1 and G-CSF. Knee Surg Sports Traumatol Arthrosc. 2013; 21:1819-1833.

5. Marmotti A, Mattia S, Perretti GM, et al. cors2013- Minced umbilical cord fragments: an effective cell source for cartilage and bone tissue engineering?- final-program.pdf. at $<$ http://www.cors2013.org/documents/cors2013-final-program.pdf>

6. Taghizadeh RR, Cetrulo KJ, Cetrulo CL. Wharton's Jelly stem cells: future clinical applications. Placenta 2011;32 Suppl 4: S311-315.

7. Marmotti A, Mattia S, Bruzzone M, et al. Minced umbilical cord fragments as a source of cells for orthopaedic tissue engineering: an in vitro study. Stem Cells Int. 2012; 2012: 326813.

8. Kim DW, Staples M, Shinozuka K, Pantcheva P, Kang SD, Borlongan CV. Wharton's jelly-derived mesenchymal stem cells: phenotypic characterization and optimizing their therapeutic potential for clinical applications. Int J Mol Sci. 2013;14:11692-11712.

9. Wu KH, Chan CK, Tsai C, et al. Effective treatment of severe steroid-resistant acute graft-versus-host disease with umbilical cord-derived mesenchymal stem cells. Transplantation. 2011; 91:1412-1416.

10. Troyer DL, Weiss ML. Wharton's jelly-derived cells are a primitive stromal cell population. Stem Cells. 2008;26:591-599.
11. Batsali AK, Kastrinaki MC, Papadaki HA, Pontikoglou C. Mesenchymal stem cells derived from Wharton's jelly of the umbilical cord: biological properties and emerging clinical applications. Curr Stem Cell Res Ther. 2013; 8:144-155.

12. Pierdomenico L. Diabetes Mellitus During Pregnancy Interferes with the Biological Characteristics of Wharton's Jelly Mesenchymal Stem Cells | StemTeCh. at <http://stemcells.unich.it/2012/11/27/diabetes-mellitus-during-pregnancy-interferes-with-the-biological-characteristics-of-whartons-jelly-mesenchymal-stem-cells/>

13. Schneider RK, Puellen A, Kramann R, et al. The osteogenic differentiation of adult bone marrow and perinatal umbilical mesenchymal stem cells and matrix remodelling in threedimensional collagen scaffolds. Biomaterials. 2010;31:467480.

14. Diao Y, Ma Q, Cui F, Zhong Y. Human umbilical cord mesenchymal stem cells: osteogenesis in vivo as seed cells for bone tissue engineering. J Biomed Mater Res A. 2009; 91:123-131.

15. Wang L, Tran I, Seshareddy K, Weiss ML, Detamore MS. A comparison of human bone marrow-derived mesenchymal stem cells and human umbilical cord-derived mesenchymal stromal cells for cartilage tissue engineering. Tissue Eng Part A. 2009; 15:2259-2266.

16. Longo UG, Loppini M, Berton A, La Verde L, Khan WS, Denaro V. Stem cells from umbilical cord and placenta for musculoskeletal tissue engineering. Curr Stem Cell Res Ther. 2012;7:272-281.

17. Leckie SK, Sowa GA, Bechara BP, et al. Injection of human umbilical tissue-derived cells into the nucleus pulposus alters the course of intervertebral disc degeneration in vivo. Spine J. 2013;13:263-272.

18. Liu S, Hou KD, Yuan M, et al. Characteristics of mesenchymal stem cells derived from Wharton's jelly of human umbilical cord and for fabrication of non-scaffold tissue-engineered cartilage. J Biosci Bioeng. 2014;117:229-235.

19. Chen X, Zhang F, He X, et al. Chondrogenic differentiation of umbilical cord-derived mesenchymal stem cells in type I collagen-hydrogel for cartilage engineering. Injury. 2013; 44:540-549.

20. Fong CY, Subramanian A, Gauthaman K, et al. Human umbilical cord Wharton's jelly stem cells undergo enhanced chondrogenic differentiation when grown on nanofibrous scaffolds and in a sequential two-stage culture medium environment. Stem Cell Rev. 2012;8:195-209. 